\title{
Geometric Modeling of Thermal Resistance in GaN HEMTs on Silicon
}

\author{
Benito González ${ }^{\circledR}$, Carlo De Santi ${ }^{\circledR}$, Fabiana Rampazzo, Matteo Meneghini ${ }^{\circledR}$, Senior Member, IEEE, \\ Antonio Nunez, Member, IEEE, Enrico Zanoni ${ }^{\circledR}$, Fellow, IEEE, \\ and Gaudenzio Meneghesso ${ }^{\mathbb{1}}$, Fellow, IEEE
}

\begin{abstract}
In this article, pulsed measurements of thermal resistance in GaN-based high-electron mobility transistors (HEMTs) on silicon, with different gate geometries and gate-to-drain extensions, are analyzed and modeled. Simple expressions for the thermal resistance of silicon-on-insulator (SOI) MOSFETs, which take into account the gate width and channel length, can be adapted to model the thermal resistance of these GaN-based HEMTs. Narrow width effects and the increase in the heat flow through the gate as the channel length increases were correctly reproduced. In addition, numerical simulations were performed to explain the reduction obtained in thermal resistance as the gate-to-drain extension increases. Our approach can also be applied easily to other wellestablished models using circuit simulators.
\end{abstract}

Index Terms-Channel temperature, electrothermal characterization, gallium nitride, high-electron mobility transistors (HEMTs), pulsed measurement, thermal resistance.

\section{INTRODUCTION}

D UE to their high breakdown voltage and power density operation, GaN-based high-electron mobility transistors (HEMTs) have become the most important devices for RF power applications, particularly following the emergence of advanced device pilot lines with silicon substrates and the consequent reductions in cost [1], [2]. When predicting the power performance of HEMTs, self-heating effects cannot be neglected [3], and this is a problem that has yet to be overcome.

Manuscript received June 29, 2020; revised August 26, 2020; accepted September 28, 2020. Date of publication October 19, 2020; date of current version November 24, 2020. This work was supported by the Ministerio de Ciencia, Innovación y Universidades (MCIU), Spain, through the Salvador de Madariaga Program, under Project PRX18/00288. The review of this article was arranged by Editor S. Graham. (Corresponding author: Benito González.)

Benito González and Antonio Nunez are with the Institute for Applied Microelectronics, Universidad de Las Palmas de Gran Canaria, 35017 Las Palmas, Spain (e-mail: benito@iuma.ulpgc.es; nunez@iuma.ulpgc.es).

Carlo De Santi, Fabiana Rampazzo, Matteo Meneghini, Enrico Zanoni, and Gaudenzio Meneghesso are with the Department of Information Engineering, University of Padova, 35131 Padova, Italy (e-mail: carlo.desanti@dei.unipd.it; rampazzo@dei.unipd.it; menego@dei.unipd.it; zanoni@dei.unipd.it; gauss@dei.unipd.it).

Color versions of one or more of the figures in this article are available online at https://ieeexplore.ieee.org.

Digital Object Identifier 10.1109/TED.2020.3028358
Several methods have been used to measure the thermal resistance (temperature rise per Watt) of GaN-based HEMTs. In a study by Kuzmík [4], a value was obtained by making use of the dc output characteristics at room temperature (subsequent current traces required the device charge to be restored) and the temperature dependences of the saturation drain current, threshold voltage, and source resistance (where the assumption of no temperature dependence for the electron saturation velocity was made). Measurements of the drain current time-domain dynamic response to positive drain bias pulses (i.e., pulsed thermal dynamic behavior) were used in [5]. Detailed temperature profiles can be created using $I R$ thermography, and in particular by high-spatial-resolution Raman thermography [6]. However, these thermography techniques are not always practical, since specific device samples and equipment are usually required in the laboratory. Finally, pulsed measurement is a feasible methodology for obtaining the thermal resistance at different ambient temperatures [3], [7]-[9], and this is the approach used in the present work.

Various studies have been carried out of the dependence on gate geometry of the measured thermal resistance of GaN-based HEMTs [4], [7], [10]. However, unlike for siliconon-insulator (SOI) MOSFETs [11]-[15], modeling of this dependence has not been extensively conducted. Most recent works are based on numerical simulations [16], [17], and have given rise to elaborated analytical closed-form expressions for thermal resistance, including device geometric parameters, different layer thicknesses, and the corresponding thermal conductivities [18], [19]. However, they do not account for implanted buffer composition, which is usually unknown and can significantly influence the self-heating of the device [20].

The use of thermal circuits that include several thermal resistances and capacitances is another option for the modeling of self-heating effects [21]. However, although detailed thermal models are desirable in order to achieve a complete physical representation, simplification is necessary when dealing with compact models, which is the main topic of this work.

The transistors under study and the experimental setup are described in Sections II and III, respectively. Section IV explains the methodology used, and the thermal resistances resulting from varying the gate geometry and gate-to-drain extension are discussed and modeled in Section V. Finally, our conclusions are presented in Section VI. 


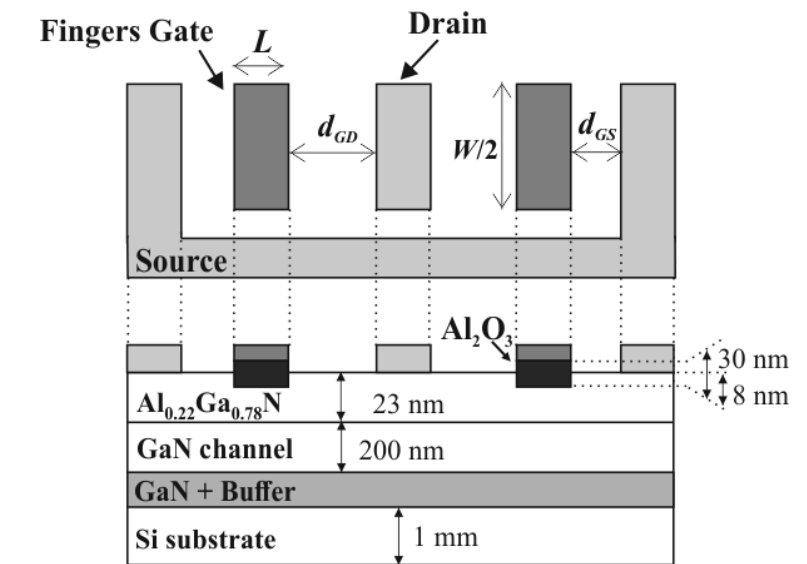

Fig. 1. Top and cross-sectional views of the device structure under study.

\section{Device Structure}

The AlGaN/GaN layer stack of the HEMTs investigated here (provided by CEA-Leti) consisted of $\mathrm{Ga}(\mathrm{Al}) \mathrm{N}$ epitaxial layers grown on a $\mathrm{Si}$ substrate of thickness $1 \mathrm{~mm}$ in the (1-1-1) direction [2], with a non-intentionally doped GaN channel and $\mathrm{Al}_{0.22} \mathrm{Ga}_{0.78} \mathrm{~N}$ barrier that were 200 and $23 \mathrm{~nm}$ thick, respectively. The depletion-mode transistors were based on a partial $\mathrm{Al}_{0.22} \mathrm{Ga}_{0.78} \mathrm{~N}$ etching, with a gate recess of $8 \mathrm{~nm}$, to give a negative threshold voltage of $-4 \mathrm{~V}$, using TiN/W (double finger) gate metal and $\mathrm{Al}_{2} \mathrm{O}_{3}$ gate oxide of thickness $30 \mathrm{~nm}$.

A reference device with a gate length, $L$, of $2 \mu \mathrm{m}$, a gate width, $W$, of $100 \mu \mathrm{m}(2 \times 50 \mu \mathrm{m})$, and a gate-to-drain extension, $d_{\mathrm{GD}}$, of $15 \mu \mathrm{m}$, was used. Other gate lengths of 1 , 3 , and $4 \mu \mathrm{m}$, total widths of 20,40 , and $200 \mu \mathrm{m}$, and gateto-drain extensions of $15,20,25$, and $30 \mu \mathrm{m}$ were also used. In all cases, the gate-to-source separation, $d_{\mathrm{GS}}$, was $2 \mu \mathrm{m}$. Top and cross-sectional views of the device structure under study, with various dimensions labeled, are shown in Fig. 1.

\section{EXPERIMENTAL Setup}

The measurement setup was performed using Agilent HP8110A and HP8114A pulse generators for gate and drain pulsing, respectively, with a pulse rise time of $10 \mathrm{~ns}$, and a Tektronix TDS680 oscilloscope [22]. The transistors were placed on a hotplate to give a temperature range of $25{ }^{\circ} \mathrm{C}-150{ }^{\circ} \mathrm{C}$ for the reverse of the substrate, which was applied in steps of $25{ }^{\circ} \mathrm{C}$. The system was remotely controlled by a personal computer using LabVIEW [3].

A diagram of the experimental setup is shown in Fig. 2. Note that in order to avoid signal reflections, a $50-\Omega$ feedthrough terminator was used to ensure efficient impedance matching between the $50-\Omega$ output pulse generator and the high-impedance gate terminal of the device under test (DUT). The drain current was determined based on Ohm's law, with the drain terminal being pulsed through a $50-\Omega$ sensing resistor, where the voltage drop was evaluated with an oscilloscope $\left(\Delta V_{\mathrm{D}}=V_{\mathrm{DD}}-V_{\mathrm{DS}}\right.$ in Fig. 2).

\section{Methodology}

The thermal resistance of the device, $R_{\mathrm{th}}$, was measured as the ratio between the channel temperature rise,

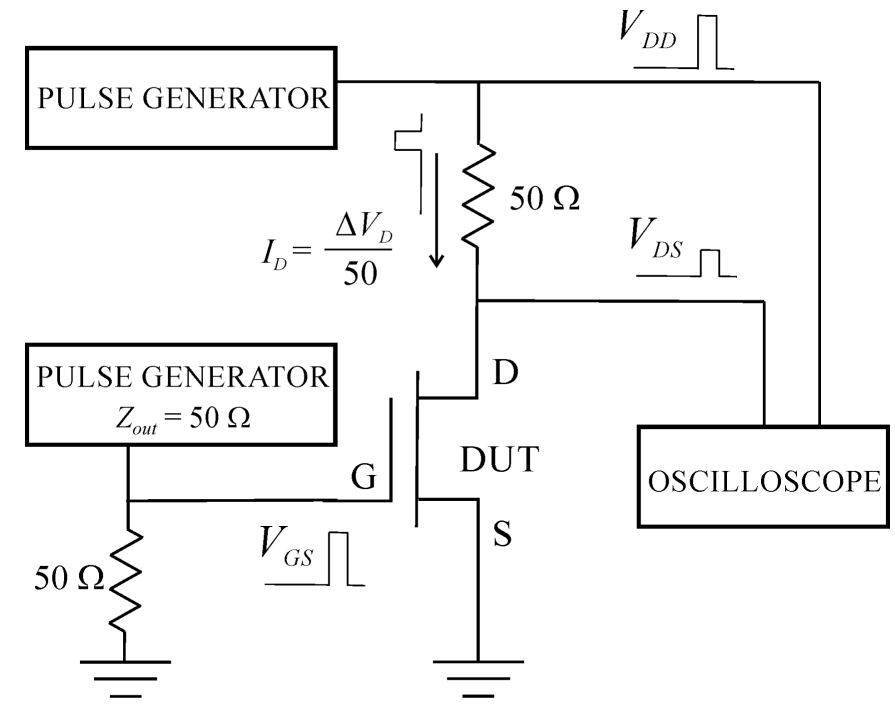

Fig. 2. Experimental setup for the double-pulsed method.

$\Delta T_{\mathrm{ch}}=T_{\mathrm{ch}}-T_{\mathrm{a}}$, with $T_{\mathrm{ch}}$ and $T_{\mathrm{a}}$ as the channel and base-plate (ambient) temperatures, respectively, and the corresponding quiescent power dissipation, $P_{\mathrm{D}}=I_{\mathrm{D}} V_{\mathrm{DS}}$, where $I_{\mathrm{D}}$ and $V_{\mathrm{DS}}$ represent the quiescent drain current and drain-source voltage, respectively (i.e., $R_{\mathrm{th}}=\Delta T_{\mathrm{ch}} / P_{\mathrm{D}}$ ), and the channel temperature rise was obtained as follows.

External heating and self-heating can modify the channel temperature of a device. First, per the methodology reported in [7], the temperature dependence of a temperature-sensitive electrical parameter (TSEP) was calibrated through external heating. In this case, the channel and base-plate (ambient) temperatures can be assumed to be equal, i.e., $T_{\mathrm{ch}} \approx T_{\mathrm{a}}$. Following this, the channel temperature under operation was obtained by measuring the same TSEP, pulsing from various bias conditions that dissipate different amounts of power, which lead to self-heating effects for a given ambient temperature.

The TSEP used as a thermometer was the maximum-pulsed drain current, $I_{\mathrm{D}, \max }$, extracted from pulsed output characteristics. In addition, to avoid disturbing the channel temperature, the pulsed voltages used should avoid any additional dynamic self-heating effects.

Thus, the output characteristics (see Fig. 3 for the reference device, with drain current per 1-mm device width) were measured using short-pulsed voltages, 200-ns wide, with a duty cycle of $0.02 \%$, from quiescent gate-to-source bias voltages without pinch-off of the channel. The current collapse was therefore minimal, since the drain-source voltage was pulsed in the ON-state [7], [22].

As previously indicated, $I_{\mathrm{D}, \max }$, for gate-to-source and drain-to-source pulsed voltages of $V_{\mathrm{GS}}=0 \mathrm{~V}$ and $V_{\mathrm{DS}}=$ $5 \mathrm{~V}$, respectively, was obtained by heating the device in two ways. First, to calibrate its temperature dependence, the ambient temperature was varied using the hotplate, with a zero power dissipation quiescent bias point, $P_{\mathrm{D}}=0 \mathrm{~W} / \mathrm{mm}$, with $V_{\mathrm{GS}}=-3 \mathrm{~V}$ and $V_{\mathrm{DD}}=0 \mathrm{~V}$, as shown in Fig. 3(a). In this case, $T_{\text {ch }} \approx T_{\mathrm{a}}=25{ }^{\circ} \mathrm{C}-150{ }^{\circ} \mathrm{C}$. Following this, as shown in Fig. 3(b), for a given ambient temperature, $T_{\mathrm{a}}$, various quiescent bias points were set with $V_{\mathrm{GS}}=0 \mathrm{~V}$ (the gate 


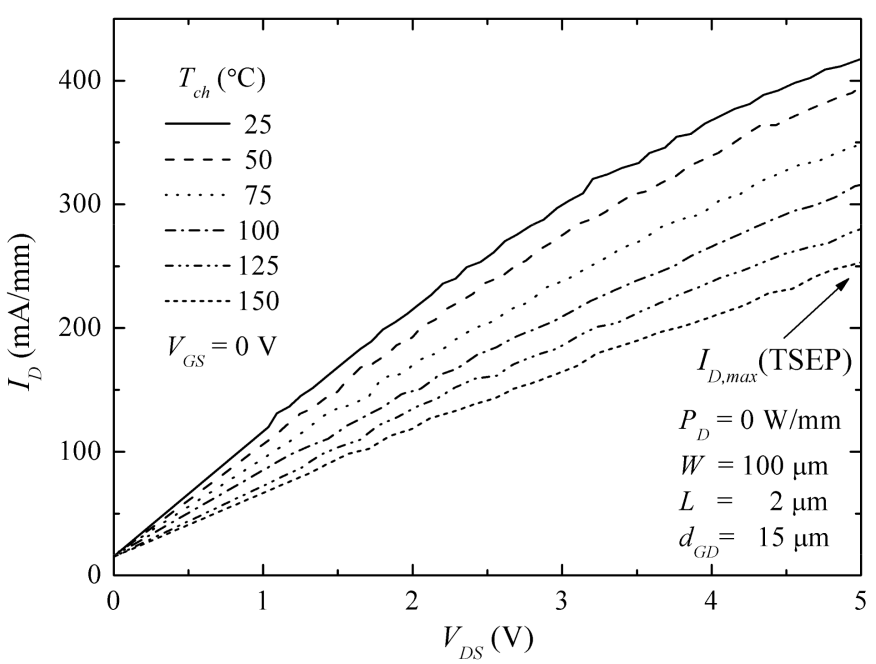

(a)

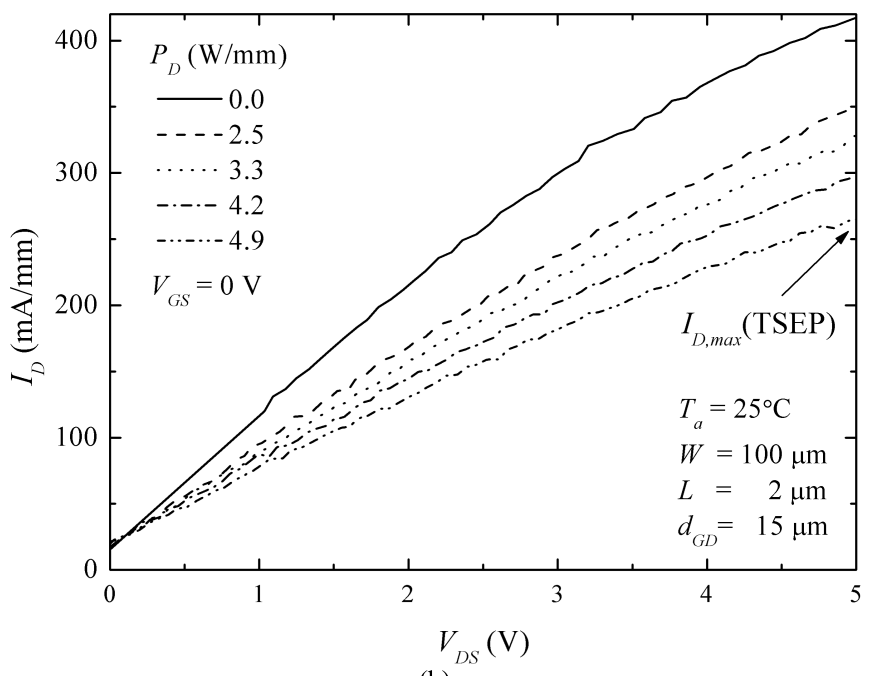

(b)

Fig. 3. Pulsed output characteristics for the reference device. (a) At different channel temperatures from zero power dissipation quiescent bias point $\left(P_{\mathrm{D}}=0 \mathrm{~W} / \mathrm{mm}\right)$. (b) From various quiescent bias points with nonzero power dissipation $\left(P_{\mathrm{D}}=0-4.9 \mathrm{~W} / \mathrm{mm}\right)$ at room temperature. $V_{\mathrm{GS}}=0 \mathrm{~V}$.

was not pulsed in this case) and different power dissipations, $P_{\mathrm{D}}=I_{\mathrm{D}} V_{\mathrm{DS}}=0-4.9 \mathrm{~W} / \mathrm{mm}$, for which $V_{\mathrm{DD}}$ was varied from 0 to $16 \mathrm{~V}$.

The maximum-pulsed drain current varied linearly with the channel temperature, as shown in Fig. 4(a) for the reference device with drain current per 1-mm device width. The measured data are represented with symbols and the corresponding linear fittings with dashed lines. At different ambient temperatures of between $25{ }^{\circ} \mathrm{C}$ and $100{ }^{\circ} \mathrm{C}, I_{\mathrm{D}, \max }$ also varied linearly with the power dissipation, as shown in Fig. 4(b). Analogous results were obtained for the remainder of the HEMTs.

Since $I_{\mathrm{D}, \max }\left(T_{\mathrm{ch}}=T_{\mathrm{a}}\right)$ and $\left.I_{\mathrm{D}, \max }\left(P_{\mathrm{D}}=0\right)\right|_{T_{\mathrm{a}}}$ coincide, the thermal resistance of the device can be evaluated as the relation between the linear deviations in the maximum-pulsed drain current with power dissipation and ambient temperature [see Fig. 4(a) and (b)], that is,

$$
R_{\mathrm{th}}\left(T_{\mathrm{a}}\right)=\frac{\Delta I_{\mathrm{D}, \max } /\left.P_{\mathrm{D}}\right|_{T_{\mathrm{a}}}}{\Delta I_{\mathrm{D}, \max } / \Delta T_{\mathrm{ch}}}
$$

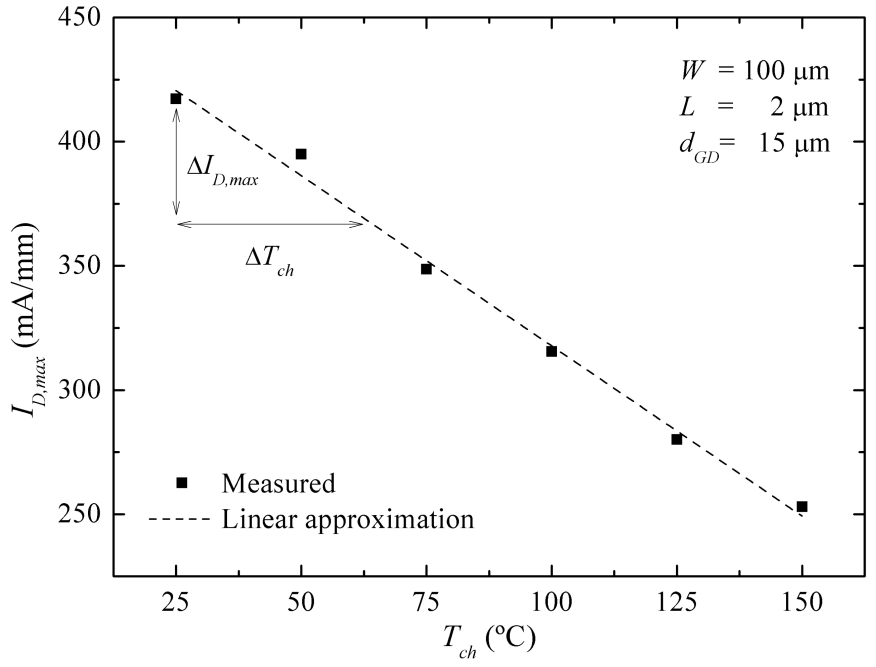

(a)

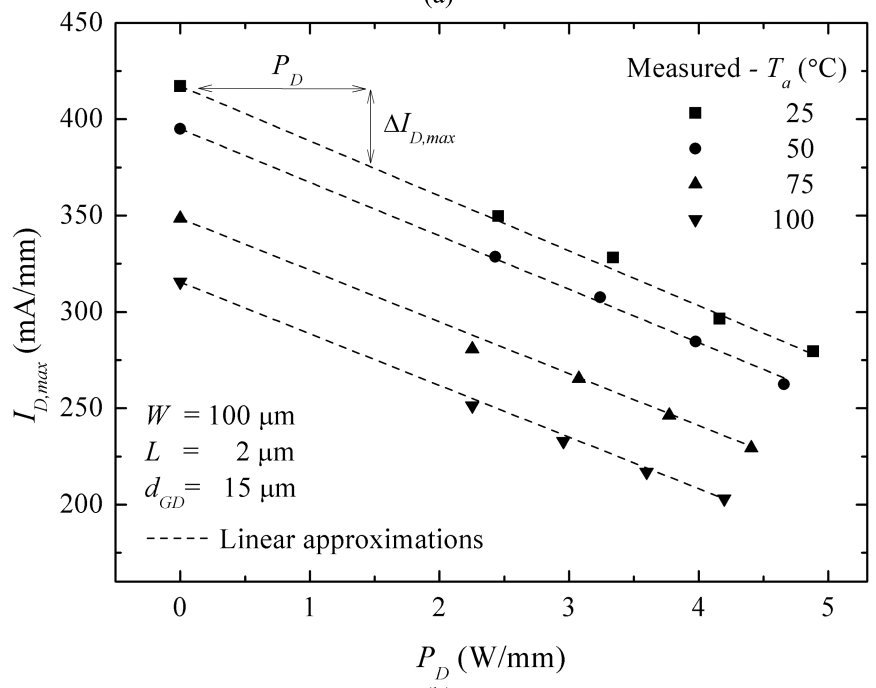

(b)

Fig. 4. Measured values of the maximum-pulsed drain current for the reference device (closed symbols) with (a) channel temperature and (b) power dissipation at different ambient temperatures. The corresponding linear approximations are represented by dashed lines. $V_{D S}=5 \mathrm{~V}$, $V_{\mathrm{GS}}=0 \mathrm{~V}$.

In this case, the thermal resistance does not depend on the power dissipation (giving linear $\Delta T_{\mathrm{ch}}-P_{\mathrm{D}}$ characteristics as a result). For the reference device, the measured thermal resistance per unit width is $19.8^{\circ} \mathrm{C} \mathrm{mm} / \mathrm{W}$, with a maximum deviation of $0.3{ }^{\circ} \mathrm{C} \mathrm{mm} / \mathrm{W}$ for the ambient temperature range used here. Similar results were obtained in [6] $\left(15.8^{\circ} \mathrm{C} \mathrm{mm} / \mathrm{W}\right)$ and [7] $\left(23.1^{\circ} \mathrm{C} \mathrm{mm} / \mathrm{W}\right)$ for double-finger GaN-based HEMTs on silicon at room temperature.

It should be pointed out that the thermal resistance of GaN-based HEMTs can increase with both power dissipation (3.9\% per Watt in [23]) and ambient temperature $(0.44 \%$ per degree Celsius in [5]). This is the case for multifinger devices if hot spot phenomena arise [6], [18], due to progressive thermal coupling as the number of fingers increases, or for HEMTs with low thermal conductivity substrates such as sapphire [24], at high power dissipation, and/or ambient temperature levels. This is attributed to a nonlinear decrease in the thermal conductivity of the semiconductors with an 
increase in the lattice temperature, which predominates over the global self-heating response of the device [25], [26]. Nonlinear $I_{\mathrm{D}, \max }-P_{\mathrm{D}}$ and $I_{\mathrm{D} \text {, } \max }-T_{\mathrm{ch}}$ characteristics can then be found to convert $I_{\mathrm{D}, \max }$ to the channel temperature [7], giving nonlinear $\Delta T_{\mathrm{ch}}-P_{\mathrm{D}}$ characteristics as a result.

\section{Measurement Results and Modeling}

In this section, we model the geometry dependence of the measured thermal resistances in the GaN-based HEMTs.

\section{A. Dependence on Gate Width}

Regarding the gate width, the thermal conductance, $G_{\text {th }}=1 / R_{\text {th }}$, obeys the linear dependence observed for SOI-MOSFETs [13], [14]. That is, $G_{\mathrm{th}}=(W+a) / R_{\mathrm{tho}}$, where $a / R_{\text {tho }}$ represents a positive thermal conductance at a hypothetical zero device width, and $R_{\text {tho }}$ represents the thermal resistance per unit width for sufficiently wide HEMTs (i.e., when $W \gg a$ and periphery effects can be neglected). The parameters $R_{\text {tho }}$ and $a$ can be determined from the slope and $W$-axis intercept, respectively [13]. Their values are shown in Table I for HEMTs with the same gate length as the reference device, $L=L_{\mathrm{ref}}=2 \mu \mathrm{m}$.

Thus, the dependence on gate width of the thermal resistance per unit width in GaN-based HEMTs is given by

$$
R_{\mathrm{th}, W}=R_{\mathrm{tho}} \frac{W}{W+a} .
$$

Hence, the wider the gate, the higher the thermal resistance per unit width, and this tends to $R_{\text {tho }}$ for a sufficiently high gate width. In this case, the thermal resistance can be expressed as the ratio between $R_{\text {tho }}$ and the gate width (i.e., $R_{\text {th }} \approx R_{\text {tho }} / W$ ), which is a common approximation. However, as mentioned above, $a$ considers the relevance of narrow width effects. For a sufficiently low gate width $(W \ll a)$, a remnant thermal resistance due to periphery effects is still present, which is given by $R_{\mathrm{th}} \approx R_{\mathrm{tho}} / a$.

Fig. 5(a) shows the measured and modeled data as symbols and a line, respectively, and good agreement between these results can be observed (with an average relative error of $10 \%) . R_{\text {tho }}$ is indicated with a dotted line. The observed narrow width effects (no constant thermal resistance per unit width) are similar to those found in [7] for SiC-based HEMTs.

\section{B. Dependence on Channel Length}

The variation in the thermal resistance per unit width with gate length was similar to that obtained in [7] and modeled as that for SOI-MOSFETs in [15]

$$
R_{\mathrm{th}, L}=R_{\mathrm{th}, W}\left[1+L_{\mathrm{o}}\left(\frac{1}{L}-\frac{1}{L_{\mathrm{ref}}}\right)\right]
$$

where $L_{\mathrm{o}}$ is a technology-dependent fitting parameter, which is shown in Table $I$, and determines the relevance of the dependence on gate length of the thermal resistance (for a null value no dependence on gate length results). Fig. 5(b) shows the measured and modeled results, represented with symbols and a line, respectively, with a relative error between them lower than $2.3 \%$.

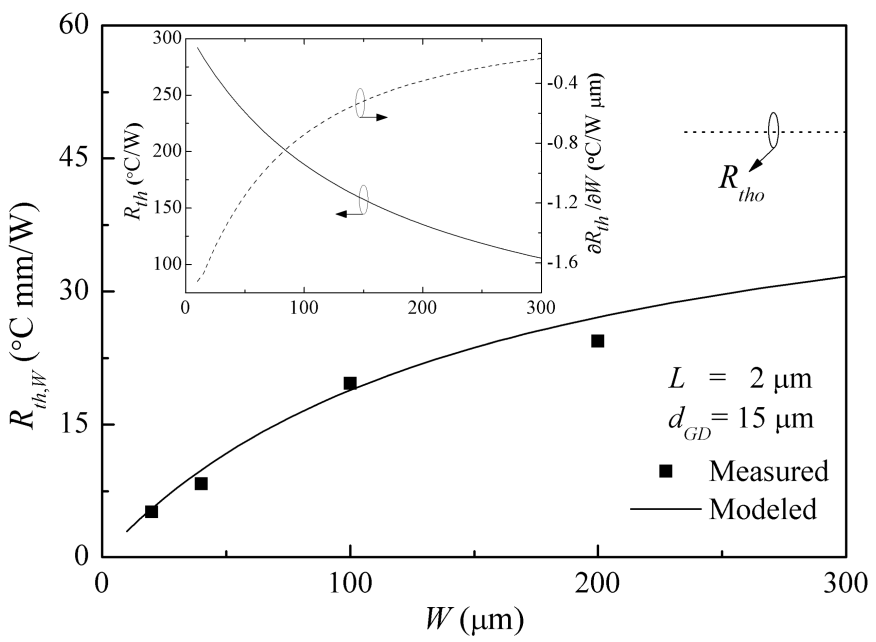

(a)

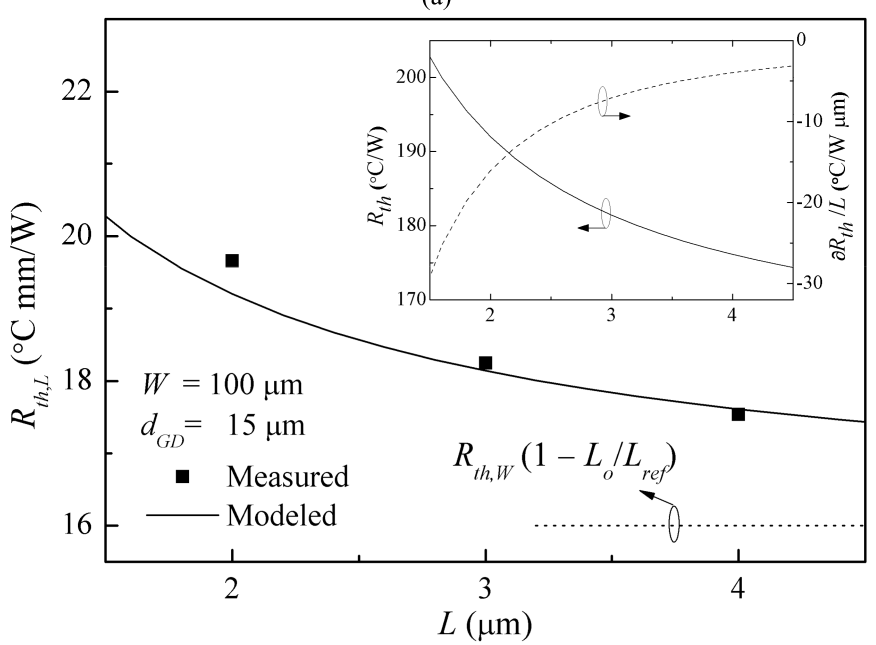

(b)

Fig. 5. Thermal resistance per unit width with (a) gate width and (b) channel length. The measured and modeled data are shown by symbols and lines, respectively. The insets show the corresponding dependence of the modeled thermal resistance (left axis, with solid line) and its derivative function (right axis, with dashed line).

TABLE I

Parameters to Model the Dependence of Thermal Resistance on Device Geometry

\begin{tabular}{ll}
\hline \hline$R_{\text {tho }}\left({ }^{\circ} \mathrm{C} \mathrm{mm} / \mathrm{W}\right)$ & 48.0 \\
$a(\mathrm{~mm})$ & 0.15 \\
$L_{o}(\mu \mathrm{m})$ & 0.33 \\
$L_{r e f}(\mu \mathrm{m})$ & 2.00 \\
$R_{t h, \infty}\left({ }^{\circ} \mathrm{C} \mathrm{mm} / \mathrm{W}\right)$ & 4.90 \\
$d_{o}(\mu \mathrm{m})$ & 43.9 \\
\hline \hline
\end{tabular}

The dependence on gate length indicates that there was a nonnegligible heat flow from channel through the gate, in contrast to the assumption, typically made for simulation purposes, that all heat flows toward the substrate [3], [17]. Further evidence of this was found in [27], where the thermal performance of a GaN HEMT on sapphire could be improved by a factor of 2.6 using a flip-chip integration approach, where bumps were placed directly onto the ohmic contacts of the source, drain, and gate. In addition, the Raman thermography temperature profiles reported in [6] demonstrated that the 
temperature at all of the terminals of a GaN-based HEMT on $\mathrm{SiC}$ (with lower thermal conductivity than $\mathrm{Si}$ ) may be significantly above the ambient temperature, indicating heat dissipation via all terminals.

In our case, the thermal resistance is reduced as the length of the channel increases, which evidences that the heat flow through the gate contact becomes more significant. However, this tendency, that increases with $L_{0}$, vanishes for very long channels [i.e., when $L \gg L_{\mathrm{ref}}$ and $R_{\mathrm{th}, L} \approx R_{\mathrm{th}, W}$ $\left(1-L_{\mathrm{o}} / L_{\mathrm{ref}}\right)=15.5^{\circ} \mathrm{C} \mathrm{mm} / \mathrm{W}$, as indicated by the dotted line in Fig. 5(b)] when no additional heat flow is dissipated via the gate. Thus, $L_{\text {ref }}$ establishes the maximum reduction in thermal resistance for very long channels.

In case a thinner $\mathrm{Al}_{2} \mathrm{O}_{3}$ gate oxide was used (note that in our case, the $30-\mathrm{nm}$ thickness is greater than that of conventional HEMTs [28]), the gate length dependence of the (reduced) thermal resistance would be enhanced.

Finally, by substituting (2) into (3), a general expression for the thermal resistance can be found, as follows:

$$
R_{\mathrm{th}}=\frac{R_{\mathrm{tho}}}{W+a}\left[1+L_{\mathrm{o}}\left(\frac{1}{L}-\frac{1}{L_{\mathrm{ref}}}\right)\right]
$$

which is simple enough to be easily applied to other wellestablished models in circuit simulators.

It should be noted that in the case of nanometric channels, where the heat flow through the gate is negligible, the thermal resistance must tend to a constant value (the source and drain contacts do not shrink) [15]. Then, in order to avoid variation in $R_{\mathrm{th}}, L+\Delta L$ should be substituted for $L$ in (3) and (4), where $\Delta L$ is a technology-dependent fitting parameter (and has a value of zero in our case).

In addition, in the case of multifinger devices, we suggest that self-heating coupling can be modeled by replacing $W$ in (2) and (4) by an effective channel width, $W_{\text {eff }}\left(W_{\text {eff }}<W\right)$, which may depend on the number of fingers, as in [14], for SOI-MOSFETs. For a physical explanation, not just for circuit simulation purposes, a more detailed model is needed.

\section{Dependence on Gate-to-Drain Extension}

Fig. 6 shows the resulting dependence on gate-to-drain extension of the measured thermal resistance per unit width (shown as closed symbols), which is similar to that obtained with the variation in gate length and is empirically modeled (shown as a line) by

$$
R_{\mathrm{th}, d_{\mathrm{GD}}}=R_{\mathrm{th}, \infty}\left(1+\frac{d_{\mathrm{o}}}{d_{\mathrm{GD}}}\right)
$$

where $d_{\mathrm{GD}}$ is the gate-to-drain extension and $d_{\mathrm{o}}$ is a technology-dependent fitting parameter that models the impact of gate-to-drain spacing on the thermal resistance, with the heat flowing from the channel, between the gate and drain, toward the gate metal, substrate, and drain terminal (as indicated below). This heat flow does not vary for very long gateto-drain spacings, when $d_{\mathrm{GD}} \gg d_{\mathrm{o}}$, and $R_{\mathrm{th}, \infty}$ represents the thermal resistance per unit width in this case. The values of these parameters are shown in Table I.

Note that the longer the gate-to-drain access region, the lower the thermal resistance results, and these tend to $R_{\mathrm{th}, \infty}$

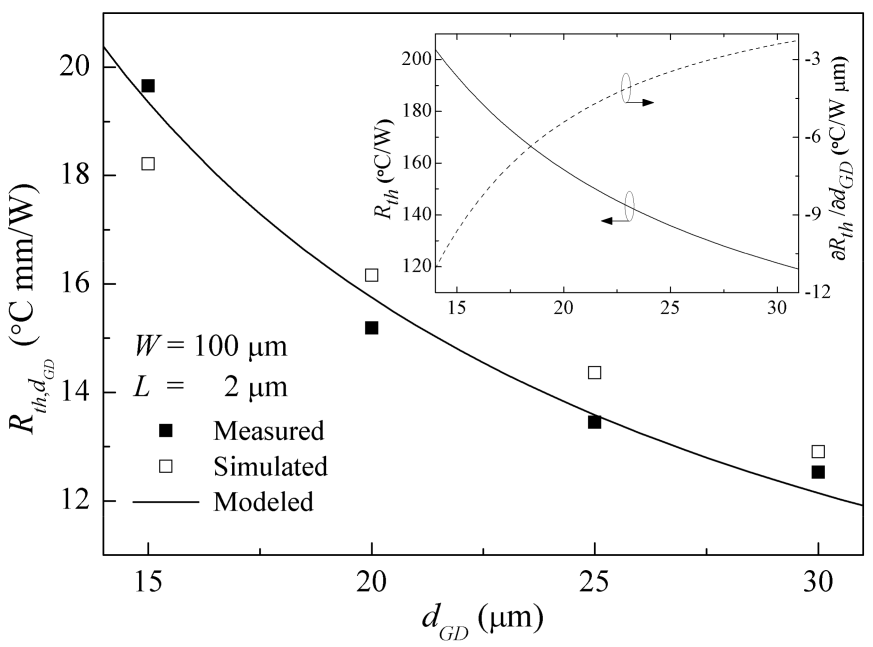

Fig. 6. Thermal resistance per unit width versus gate-to-drain extension. The measured, simulated, and modeled data are shown by the closed symbols, open symbols, and line, respectively. The inset shows the corresponding dependence of the modeled thermal resistance (left axis, with solid line) and its derivative function (right axis, with dashed line).

only after several millimeters (an impracticable size). The reduction in heat flow through the drain contact, as the gate-to-drain extension increases, would lead to an increase in the thermal resistance (in our case, metallization of the source/drain contacts extended down to the channel, acting as an efficient heat sink). The opposite behavior of the thermal resistance observed in this experiment must therefore be due to a larger increase in the dissipation of internal heat to other parts of the device. In order to clarify this point, 2-D numerical simulations were performed with Sentaurus Device [29] by solving the heat flow equation with the Poisson and drift-diffusion equations. Due to symmetry, only half of the device needed to be simulated. The polarization charges and saturation velocity effects were included. Dirichlet boundary conditions for the lattice temperature $\left(25^{\circ} \mathrm{C}\right)$ and proper surface thermal resistances $\left(0.009{ }^{\circ} \mathrm{C} \mathrm{cm}^{2} / \mathrm{W}\right)$ were used at all terminals, and the substrate was replaced by an additional equivalent surface thermal resistance $\left(0.006{ }^{\circ} \mathrm{C} \mathrm{cm}^{2} / \mathrm{W}\right)$. Thus, the obtained gate-to-drain extension dependence of the thermal resistance was numerically reproduced, as shown by the open symbols in Fig. 6, with a relative error between the measured and simulated data of less than $7.5 \%$ in all cases.

Fig. 7 shows the resulting temperature profile for the reference device (half of the device is shown with relevant geometric dimensions). The inset shows a magnified view of the gate area with field plates G-FP1 and G-FP2, located above the thin film of silicon nitride (thickness $150 \mathrm{~nm}$ ) grown on the $\mathrm{AlGaN}$ barrier and the $\mathrm{SiO}_{2}$ field oxide (thickness $400 \mathrm{~nm}$ ), respectively. Similar results were obtained for the remainder of the devices. The results indicate that a significant heat flow is not only dissipated from the device via the substrate and the reverse of the contacts, but also the field plates, and particularly the gate field plates, via the passivation layer/barrier interface. It is known that the hottest region in the channel extends outward into the drain extension below G-FP1 [6], and this could be increased by the velocity overshoot effect [30]. The increase in the simulated heat flow through the gate 


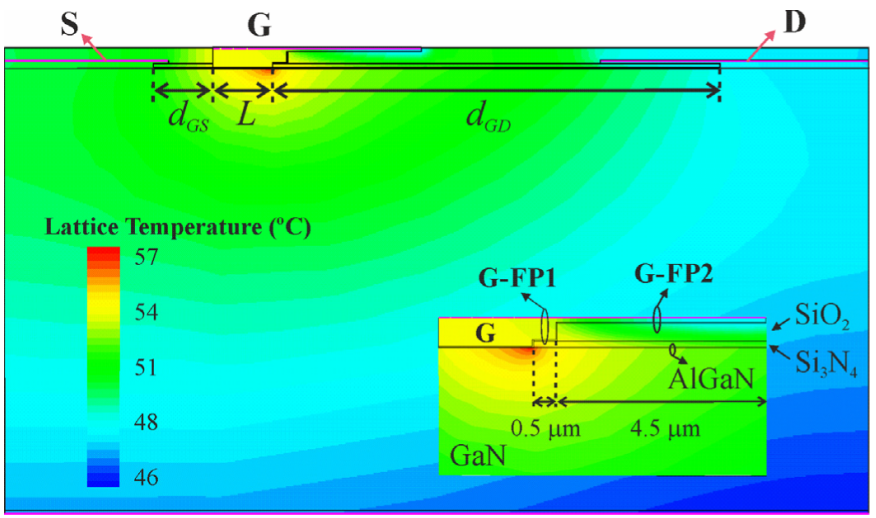

Fig. 7. Lattice temperature profile for the reference device (half of the device is shown here). The inset shows a magnified view of the gate area with the gate field plates (G-FP1 and G-FP2). $V_{\mathrm{DS}}=5 \mathrm{~V}, V_{\mathrm{GS}}=0 \mathrm{~V}$, $T_{\mathrm{a}}=25^{\circ} \mathrm{C}$.

metal and substrate as the gate-to-drain extension is increased predominates over the heat flow reduction toward the drain, giving rise to the gate-to-drain dependence of the thermal resistance as shown in Fig. 6.

The gate-to-drain extension is a technology-dependent parameter that cannot be controlled by circuit designers. High values of this parameter are desirable in order to reduce the thermal resistance of the device. However, this may negatively affect other necessary device performance characteristics, such as a high current supply and/or low ON-resistance, $R_{\text {on }}$. A suitable value for $d_{\mathrm{GD}}$ is required in a given application.

For the suitable gate-to-drain extension, the thermal resistance can be determined by following the procedure described in Sections V-A and V-B.

Finally, for a better comprehension of the dependence on gate width, gate length, and gate-to-drain extension of the thermal resistance, the insets in Figs. 5(a) and (b) and 6 show the corresponding dependence of the modeled thermal resistance (left axis, with solid line) and its derivative function (right axis, with dashed line). Notice that, as expected in all cases, the thermal resistance significantly reduces as the device geometry expands since more heat flow can be spread out of the device through the terminals (including field-plates) and substrate. For the same variation in gate width, gate length, and gate-to-drain extension, the dependence on gate length and gate width turns out to be the most and least relevant, respectively. Nevertheless, compared with the changes in gate length and gate-to-drain extension, the change in gate width is much greater (it could be up to several millimeters). Therefore, in practice, the channel width has a more significant impact on device self-heating.

\section{CONCLUSION}

The pulsed methodology described here is a well-established approach that was successfully applied to determine the thermal resistance of GaN-based HEMTs for different gate geometries and gate-to-drain access regions. The expected dependencies of the thermal resistance on channel width and gate length were observed and successfully modeled using simple expressions previously applied to SOI-MOSFETs. In this way, narrow width effects were correctly reproduced, and the increase in the heat flow through the gate as the channel length increases was derived by a reduction of the thermal resistance, which vanishes with a channel length of greater than a few microns. Additional numerical simulations demonstrated that the increase in the thermal flow through the gate metal and substrate as the gate-to-drain extension increases predominates over the reduction toward the drain contact, giving rise to a reduction in the thermal resistance, which was also modeled. Finally, the modeling approach developed here to take into account the self-heating effects can be easily applied to circuit simulators as an add-on to other well-established models.

\section{REFERENCES}

[1] H. Amano et al., "The $2018 \mathrm{GaN}$ power electronics roadmap," J. Phys. D, Appl. Phys., vol. 51, no. 16, Apr. 2018, Art. no. 163001, doi: 10.1088/1361-6463/aaaf9d.

[2] L. Di Cioccio et al., "From epitaxy to converters topologies what issues for $200 \mathrm{~mm}$ GaN/Si?" in IEDM Tech. Dig., Washington, DC, USA, Dec. 2015, p. 16, doi: 10.1109/IEDM.2015.7409712.

[3] A. Chini, F. Soci, M. Meneghini, G. Meneghesso, and E. Zanoni, "Deep levels characterization in GaN HEMTs-Part II: Experimental and numerical evaluation of self-heating effects on the extraction of traps activation energy," IEEE Trans. Electron Devices, vol. 60, no. 10, pp. 3176-3182, Oct. 2013, doi: 10.1109/TED.2013.2278290.

[4] J. Kuzmik, R. Javorka, A. Alam, M. Marso, M. Heuken, and P. Kordos, "Determination of channel temperature in AlGaN/GaN HEMTs grown on sapphire and silicon substrates using DC characterization method," IEEE Trans. Electron Devices, vol. 49, no. 8, pp. 1496-1498, Aug. 2002, doi: 10.1109/TED.2002.801430.

[5] C. Florian, A. Santarelli, R. Cignani, and F. Filicori, "Characterization of the nonlinear thermal resistance and pulsed thermal dynamic behavior of AlGaN-GaN HEMTs on SiC," IEEE Trans. Microw. Theory Techn., vol. 61, no. 5, pp. 1879-1891, May 2013, doi: 10.1109/TMTT.2013.2256146.

[6] M. Kuball and J. W. Pomeroy, "A review of Raman thermography for electronic and opto-electronic device measurement with submicron spatial and nanosecond temporal resolution," IEEE Trans. Device Mater. Rel., vol. 16, no. 4, pp. 667-684, Dec. 2016, doi: 10.1109/TDMR.2016.2617458.

[7] J. Joh, J. A. del Alamo, U. Chowdhury, T.-M. Chou, H.-Q. Tserng, and J. L. Jimenez, "Measurement of channel temperature in GaN high-electron mobility transistors," IEEE Trans. Electron Devices, vol. 56, no. 12, pp. 2895-2901, Dec. 2009, doi: 10.1109/TED.2009.2032614.

[8] N. Killat, M. Kuball, T.-M. Chou, U. Chowdhury, and J. Jimenez, "Temperature assessment of AlGaN/GaN HEMTs: A comparative study by Raman, electrical and IR thermography," in Proc. IEEE Int. Rel. Phys. Symp., Anaheim, CA, USA, May 2010, pp. 528-531.

[9] R. Sommet, G. Mouginot, R. Quere, Z. Ouarch, and M. Camiade, "Thermal modeling and measurements of AlGaN/GaN HEMTs including thermal boundary resistance," Microelectron. J., vol. 43, no. 9 , pp. 611-617, Sep. 2012, doi: 10.1016/j.mejo.2011.07.009.

[10] R. Rodríguez, B. González, J. García, and A. Núñez, "Electrothermal DC characterization of GaN on Si MOS-HEMTs," Solid-State Electron., vol. 137, pp. 44-51, Nov. 2017, doi: 10.1016/j.sse.2017.08.002.

[11] C. Anghel, R. Gillon, and A. M. Ionescu, "Self-heating characterization and extraction method for thermal resistance and capacitance in HV MOSFETs," IEEE Electron Device Lett., vol. 25, no. 3, pp. 141-143, Mar. 2004, doi: 10.1109/LED.2003.821669.

[12] S. Makovejev, S. H. Olsen, V. Kilchytska, and J.-P. Raskin, "Time and frequency domain characterization of transistor self-heating," IEEE Trans. Electron Devices, vol. 60, no. 6, pp. 1844-1851, Jun. 2013, doi: 10.1109/TED.2013.2259174.

[13] S. Lee et al., "Experimental analysis and modeling of self heating effect in dielectric isolated planar and fin devices," in Proc. IEEE Symp. VLSI Technol., Kyoto, Japan, Jun. 2013, pp. T248-T249.

[14] B. González, R. Rodriguez, and A. Lazaro, "Thermal resistance characterization for multifinger SOI-MOSFETs," IEEE Trans. Electron Devices, vol. 65, no. 9, pp. 3626-3632, Sep. 2018, doi: 10.1109/TED.2018.2853799.

[15] P. Kushwaha et al., "Thermal resistance modeling in FDSOI transistors with industry standard model BSIM-IMG," Microelectron. J., vol. 56, pp. 171-176, Oct. 2016, doi: 10.1016/j.mejo.2016.07.014. 
[16] A. Chvala, J. Marek, P. Pribytny, A. Satka, M. Donoval, and D. Donoval, "Effective 3-D device electrothermal simulation analysis of influence of metallization geometry on multifinger power HEMTs properties," IEEE Trans. Electron Devices, vol. 64, no. 1, pp. 333-336, Jan. 2017, doi: 10.1109/TED.2016.2629024.

[17] A. K. Sahoo et al., "Thermal analysis of AlN/GaN/AlGaN HEMTs grown on $\mathrm{Si}$ and $\mathrm{SiC}$ substrate through TCAD simulations and measurements," in Proc. 11th Eur. Microw. Integr. Circuits Conf. (EuMIC), London, U.K., Oct. 2016, pp. 145-148, doi: 10.1109/EuMIC.2016.7777511.

[18] A. Darwish, A. J. Bayba, and H. A. Hung, "Channel temperature analysis of GaN HEMTs with nonlinear thermal conductivity," IEEE Trans. Electron Devices, vol. 62, no. 3, pp. 840-846, Mar. 2015, doi: 10.1109/TED.2015.2396035.

[19] Y. Jia, Y. Xu, and Y. Guo, "A universal scalable thermal resistance model for compact large-signal model of AlGaN/GaN HEMTs," IEEE Trans. Microw. Theory Techn., vol. 66, no. 10, pp. 4419-4429, Oct. 2018, doi: 10.1109/TMTT.2018.2854185.

[20] J. Kuzmik et al., "Self-heating in GaN transistors designed for highpower operation," IEEE Trans. Electron Devices, vol. 61, no. 10, pp. 3429-3434, Oct. 2014, doi: 10.1109/TED.2014.2350516.

[21] C. Wang et al., "An electrothermal model for empirical Large- signal modeling of $\mathrm{AlGaN} / \mathrm{GaN}$ HEMTs including self-heating and ambient temperature effects," IEEE Trans. Microw. Theory Techn., vol. 62, no. 12, pp. 2878-2887, Dec. 2014, doi: 10.1109/TMTT.2014.2364821.

[22] G. Meneghesso et al., "Surface-related drain current dispersion effects in AlGaN-GaN HEMTs,' IEEE Trans. Electron Devices, vol. 51, no. 10, pp. 1554-1561, Oct. 2004, doi: 10.1109/TED.2004.835025.
[23] J. Liu, L. Sun, Z. Yu, and M. Condon, "A simple method to determine power-dissipation dependent thermal resistance for GaN HEMTs," in IEEE MTT-S Int. Microw. Symp. Dig., Baltimore, MD, USA, Jun. 2011, pp. 1-4, doi: 10.1109/MWSYM.2011.5972752.

[24] R. Menozzi et al., "Temperature-dependent characterization of AlGaN/GaN HEMTs: Thermal and Source/Drain resistances," IEEE Trans. Device Mater. Rel., vol. 8, no. 2, pp. 255-264, Jun. 2008, doi: 10.1109/TDMR.2008.918960.

[25] J. Piprek, Semiconductor Optoelectronic Devices: Introduction to Physics and Simulation. San Diego, CA, USA: Academic, 2001, p. 22.

[26] H. Sun et al., "Temperature-dependent thermal resistance of GaN-ondiamond HEMT wafers," IEEE Electron Device Lett., vol. 37, no. 5, pp. 621-624, May 2016, doi: 10.1109/LED.2016.2537835.

[27] J. Das et al., "Improved thermal performance of AlGaN/GaN HEMTs by an optimized flip-chip design," IEEE Trans. Electron Devices, vol. 53, no. 11, pp. 2696-2702, Nov. 2006, doi: 10.1109/TED.2006. 883944.

[28] G. Dutta, N. DasGupta, and A. DasGupta, "Effect of sputtered- $\mathrm{Al}_{2} \mathrm{O}_{3}$ layer thickness on the threshold voltage of III-nitride MIS-HEMTs,", IEEE Trans. Electron Devices, vol. 64, no. 4, pp. 1450-1458, Apr. 2016, doi: 10.1109/TED.2016.2529428.

[29] Sentaurus Device User Guide, Version N-2017.09, Synopsys, Mountain View, CA, USA, 2019.

[30] B. González, B. Iñiguez, A. Lázaro, and A. Cerdeira, "Numerical DC self-heating in planar double-gate MOSFETs," Semicond. Sci. Technol., vol. 26, no. 9, Sep. 2011, Art. no. 095014, doi: 10.1088/0268$1242 / 26 / 9 / 095014$. 